\title{
Angular distribution and azimuthal asymmetry for pentaquark production in proton-proton collisions
}

\author{
H.W. Barz \\ Institut für Kern- und Hadronenphysik, Forschungszentrum Rossendorf, Pf 510119, D-01314 Dresden, Germany \\ M. Zétényi \\ KFKI Research Institute for Particle and Nuclear Physics, POB 49, H-1525 Budapest, Hungary
}

(Dated: July 4, 2018)

\begin{abstract}
Angular distributions for production of the $\Theta^{+}$pentaquark are calculated for the collisions of polarized protons with polarized target protons. We compare calculations based on different assumptions concerning spin and parity $\left(J=1 / 2^{ \pm}, 3 / 2^{ \pm}\right)$of the $\Theta^{+}$state. For a wide class of interactions the spin correlation parameters describing the asymmetric angular distributions are calculated up to $250 \mathrm{MeV}$ above production threshold. The deviations from the near threshold behavior are investigated.
\end{abstract}

PACS numbers: $12.39 . \mathrm{Mk}, 13.75 . \mathrm{Cs}, 13.88 .+\mathrm{e}, 14.20 .-\mathrm{c}$

\section{INTRODUCTION}

Recently a number of experiments $1,2,[3,4,5,6,7]$ have confirmed the existence of a narrow pentaquark state $\Theta^{+}$ with a mass of about $1.53 \mathrm{GeV}$. It decays into the $p K^{0}$ or the $n K^{+}$channel with a width of less than about $15 \mathrm{MeV}$. Its positive strangeness $S=+1$ and the small width give good reason to identify this resonance with the pentaquark state predicted in ref. [8] within the chiral soliton model. According to this approach it belongs to a $J^{\pi}=1 / 2^{+}$ antidecuplet as an isospin singlet with the five quark configuration uudds . However, the spin-parity assignment is experimentally still not verified. First measurements are done to observe this resonance in proton-proton collision $p p \rightarrow p \Sigma^{+} K^{0}[9]$ at near threshold energy where the invariant masses of the $p K^{0}$ pair indicate the existence of the $\Theta^{+}$resonance.

Several investigations are made to find observables to determine spin and parity. In photo-production processes the cross sections were studied using $K\left[10,11\right.$ and $K$ and $K^{*}$ 12, 13 exchange Lagrangians. For nucleon-nucleon reactions the cross section [1] and the azimuthal angular distribution [14, 15. were analyzed. Especially in the near threshold region the azimuthal angular distribution is very sensitive to distinguish between different parity assignments of the $\Theta^{+}$particle. The great advantage of using the threshold region lies in the fact that to a large extent model independent predictions can be made [14, 16, 17, 18].

In this work we are going to extend the study of proton-proton reactions to larger energies using a model dependent interaction. We employ a combination of $K$ and $K^{*}$ exchange, where the parameters are constraint by assuming a production cross section as was found in the experiment [9]. The azimuthal angular distribution is parametrized by correlation coefficients, which we calculate assuming four different spin-parity states $1 / 2^{ \pm}, 3 / 2^{ \pm}$of the $\Theta^{+}$resonance. It is the aim to investigate how the threshold behavior of the azimuthal angular distribution changes if the energy is increased above threshold.

\section{THE MODEL}

The simplest mechanism than can be used to describe the reaction $p p \rightarrow \Sigma^{+} \Theta^{+}$is the exchange of a pseudoscalar kaon given by the interaction Lagrangian

$$
\begin{aligned}
& \mathcal{L}_{K N \Theta}=i g_{K N \Theta} \overline{\Theta^{+}} \gamma^{5}\left(p K^{0}+n K^{+}\right)+\text {h.c. } \\
& \mathcal{L}_{K N \Sigma}=i \sqrt{2} g_{K N \Sigma} \overline{\Sigma^{+}} \gamma^{5}\left(p \overline{K^{0}}+n \overline{K^{+}}\right)+\text {h.c. }
\end{aligned}
$$

for the $1 / 2^{+}$state of the $\Theta^{+}$particle. The symbols $\Theta^{+}, \Sigma^{+}, p, n$ stand for the spinors of the participating Fermions and $K^{ \pm}$for the bosonic wave functions. The factor $\sqrt{2}$ in Eq. (2) comes from the isospin factor in the standard representation [19]. The coupling constant $g_{K N \Theta}$ is related to the decay width of the $\Theta^{+}$into to the $K^{+}$and $K^{0}$ channels via

$$
\Gamma_{\Theta}=g_{K N \Theta}^{2} \frac{p_{N}\left(p_{N}^{0}-m_{N}\right)}{2 \pi m_{\Theta}}
$$


TABLE I: Coupling strengths and cut-off parameters assuming two different decay widths $\Gamma_{\Theta}$. The last two columns give the positive and negative limits of the coupling constants for the $K^{*}$ exchange in Eqs. (1112) which do not increase the cross section by more than a factor of two.

\begin{tabular}{|c||c|c||c|c|c|c|}
\hline \multicolumn{1}{|c||}{$J^{\pi}$} & \multicolumn{2}{c||}{$\Gamma_{\Theta}=1 \mathrm{MeV}$} & \multicolumn{4}{c|}{$\Gamma_{\Theta}=10 \mathrm{MeV}$} \\
& $\mathrm{g}(\mathrm{f})$ & $\Lambda(\mathrm{GeV})$ & $\mathrm{g}(\mathrm{f})$ & $\Lambda(\mathrm{GeV})$ & $g^{*}\left(f^{*}\right)$ \\
\hline $1 / 2^{+}$ & 1.03 & 1.03 & 3.27 & 0.76 & 0.3 & -0.6 \\
$1 / 2^{-}$ & 0.14 & 1.55 & 0.44 & 0.94 & 0.6 & -0.6 \\
$3 / 2^{+}$ & 0.46 & 0.90 & 1.47 & 0.70 & 2.0 & -0.9 \\
$3 / 2^{-}$ & 3.44 & 0.69 & 10.9 & 0.60 & 3.1 & -3.5 \\
\hline
\end{tabular}

where $p_{N}$ denotes the momentum of the emitted proton with $p_{N}^{0}$ being the energy as the zeroth component. Assuming a width of $10 \mathrm{MeV}$ and a mass of $\Theta^{+}$of $m_{\Theta}=1.53 \mathrm{GeV}$ we obtain $g_{K N \Theta}=3.27$. (Another possible approach for the determination of $g_{K N \Theta}$ uses SU(3) relations of the pentaquark multiplets [20]. Assuming that the N(1710) baryon resonance is an ideal mixture of antidecuplet and octet pentaquark states, they obtain $g_{K N \Theta}=3.0$ [21].) The coupling constant $g_{K N \Sigma}$ was estimated within the framework of SU(3) [1, 19] to be $g_{K N \Sigma}=-3.78$. (The actual value depends somewhat on the data the SU(3) parameters are adjusted to.) Furthermore we use a monopol formfactor

$$
F\left(q^{2}\right)=\frac{\Lambda^{2}-m_{K}^{2}}{\Lambda^{2}-q^{2}}
$$

with $q$ being the square of the transferred four-momentum.

In the following we also consider the possibilities that spin and parity of the $\Theta^{+}$could take the values $J^{\pi}=1 / 2^{-}$, $3 / 2^{+}$, or $3 / 2^{-}$. The corresponding Lagrangians in their simplest form read

$$
\begin{aligned}
\mathcal{L}^{\prime}{ }_{K N \Theta} & =g_{K N \Theta}^{\prime} \overline{\Theta^{+}}\left(p K^{0}+n K^{+}\right)+\text {h.c. }, \\
\mathcal{L}^{\prime \prime}{ }_{K N \Theta} & =\frac{f_{K N \Theta}}{m_{K}} \overline{\Theta_{\mu}^{+}}\left(p \partial^{\mu} K^{0}+n \partial^{\mu} K^{+}\right)+\text {h.c. }, \\
\mathcal{L}^{\prime \prime \prime}{ }_{K N \Theta} & =i \frac{f_{K N \Theta}^{\prime}}{m_{K}} \overline{\Theta_{\mu}^{+}} \gamma^{5}\left(p \partial^{\mu} K^{0}+n \partial^{\mu} K^{+}\right)+\text {h.c. },
\end{aligned}
$$

where $\Theta_{\mu}^{+}$is the Rarita-Schwinger representation of a spin $3 / 2$ state. The coupling constant $g^{\prime}, f, f^{\prime}$ are related to the width via

$$
\begin{aligned}
\Gamma_{\Theta} & =g_{K N \Theta}^{\prime 2} \frac{p_{N}\left(p_{N}^{0}+m_{N}\right)}{2 \pi m_{\Theta}} \\
& =f_{K N \Theta}^{2} \frac{p^{3}\left(p_{N}^{0}+m_{N}\right)}{6 \pi m_{K}^{2} m_{\Theta}} \\
& =f_{K N \Theta}^{\prime 2} \frac{p^{3}\left(p_{N}^{0}-m_{N}\right)}{6 \pi m_{K}^{2} m_{\Theta}}
\end{aligned}
$$

leading to the values given in Table 【

Also other processes can contribute to the production. As an example we include the $K^{*}$ exchange in addition to the $\mathrm{K}$ exchange. For the positive parity state of a spin $1 / 2$ baryon $B\left(\Sigma\right.$ or $\left.\Theta^{+}\right)$we use

$$
\mathcal{L}_{1 / 2^{+}}=g_{K^{*} N B}^{*} \bar{B}\left(\gamma^{\mu}+\frac{\kappa}{m_{\Theta}+m_{N}} \sigma^{\nu \mu} \partial_{\nu}\right) K_{\mu}^{0 *} p+\text { h.c. }
$$

and for the $3 / 2^{+}$state

$$
\mathcal{L}_{3 / 2^{+}}=i \frac{f_{K^{*} N \Theta}^{*}}{m_{K^{*}}} \overline{\Theta^{+\mu}} \gamma^{5} \gamma^{\nu} p\left(\partial_{\nu} K_{\mu}^{0 *}-\partial_{\mu} K_{\nu}^{0 *}\right)+h . c .
$$

For the negative parity states we insert the factor $i \gamma^{5}$ into Eq. (11) and remove this factor from Eq. (12). The coupling constants for the $\Sigma N$ coupling in Eq. (11) $g_{K^{*} N \Sigma}^{*}=-3.25 \sqrt{2}$ and $\kappa=1.8$ are chosen in accordance with [11, 19]. For the $\Theta^{+}$particle the coupling constants will be fixed later. 


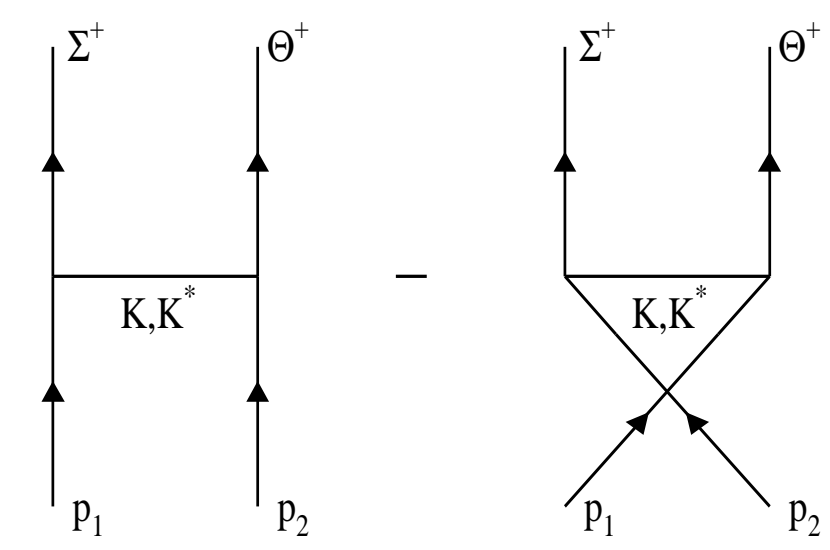

FIG. 1: Calculated diagrams for $\Theta^{+}$production in a $p p$ collision.

Accordingly to the diagrams shown in Fig. 10 we calculate the T matrix from which the differential cross sections is calculated in the center-of-mass system

$$
\frac{d \sigma}{d \Omega}=\frac{p_{\Theta}}{64 \pi^{2} s p_{1}} \frac{1}{4} \sum_{\text {spins }} T_{s_{1}, s_{2}, s_{\Sigma}, s_{\Theta}}^{*}\left(1+\mathbf{P}_{1} \vec{\sigma}_{1}\right)\left(1+\mathbf{P}_{2} \vec{\sigma}_{2}\right) T_{s_{1}, s_{2}, s_{\Sigma}, s_{\Theta}}
$$

as a function of the incoming momentum $p_{1}$, the outgoing momentum $p_{\Theta}$ and the center-of-mass energy $\sqrt{s}$. The polarization of the incoming (target) proton is described by the vector $\mathbf{P}_{1(2)}$, and $\vec{\sigma}_{1(2)}$ is the Pauli matrix which acts on the first (second) spin index, respectively.

For convenience we choose our coordinate system such that the z-axis coincides with the direction of the incoming proton. The $\mathrm{y}$-axis is chosen orthogonal to the reaction plane in the direction of the normal vector $\mathbf{p}_{\mathbf{1}} \times \mathbf{p}_{\boldsymbol{\Theta}}$ and the $\mathrm{x}$-axis points to the side direction of $\mathbf{n} \times \mathbf{p}_{\mathbf{1}}$. From general considerations [22, 23. one can show that the differential cross section can only be a function of the following combinations of the polarization vectors

$$
\begin{aligned}
\frac{d \sigma}{d \Omega}= & \left(\frac{d \sigma}{d \Omega}\right)_{0}\left(1+A_{y 0} P_{1 y}+A_{0 y} P_{2 y}+A_{y y} P_{1 y} P_{2 y}+A_{x x} P_{1 x} P_{2 x}\right. \\
& \left.+A_{x z} P_{1 x} P_{2 z}+A_{z x} P_{1 z} P_{2 x}+A_{z z} P_{1 z} P_{2 z}\right) .
\end{aligned}
$$

Here the symbols $P_{x}, P_{y}, P_{z}$ stand for the components of the polarization vectors $\mathbf{P}_{\mathbf{1}(\mathbf{2})}$. The first factor in Eq. (14) is the differential cross section for unpolarized protons. The coefficients $A$ depend on the polar angle $\Theta$.

Splitting the polarization vectors into components parallel and perpendicular with respect to the beam direction we rewrite Eq. (14) as a function of the azimuth angle $\phi$ as

$$
\begin{aligned}
\frac{d \sigma}{d \Omega}=\left(\frac{d \sigma}{d \Omega}\right)_{0}(1 & +A_{y 0} P_{1 \perp} \sin \left(\phi-\alpha_{1}\right)+A_{0 y} P_{2 \perp} \sin \left(\phi-\alpha_{2}\right) \\
& +A_{y y} P_{1 \perp} P_{2 \perp} \sin \left(\phi-\alpha_{1}\right) \sin \left(\phi-\alpha_{2}\right) \\
& +A_{x x} P_{1 \perp} P_{2 \perp} \cos \left(\phi-\alpha_{1}\right) \cos \left(\phi-\alpha_{2}\right) \\
& +A_{z z} P_{1 z} P_{2 z} \\
& \left.+A_{x z} P_{1 \perp} P_{2 z} \cos \left(\phi-\alpha_{1}\right)+A_{z x} P_{1 z} P_{2 \perp} \cos \left(\phi-\alpha_{2}\right)\right) .
\end{aligned}
$$

Here $\alpha_{1}, \alpha_{2}$ denote the angles of the projections of the polarization vectors onto the $\mathrm{x}$-y plane. The coefficients $A_{i j}$ are usually called spin correlation parameters which depend on the azimuth angle $\phi$ only up to second order in $\cos (\phi)$ or $\sin (\phi)$. Since the initial state is symmetric with respect to the z-axis the parameters $A_{x x}, A_{y y}, A_{z z}$ are forward-backward symmetric while the others obey the relations

$$
\begin{aligned}
& A_{z x}(\Theta)=-A_{x z}(\pi-\Theta) \\
& A_{0 y}(\Theta)=-A_{y 0}(\pi-\Theta)
\end{aligned}
$$

similar to those for $p p \rightarrow p p \pi$ collisions [23]. In our calculations using lowest order perturbation theory the coefficient $A_{y 0}$ vanishes. Thus, we do not obtain azimuthal asymmetry if the proton in the beam or in the target is unpolarized. 


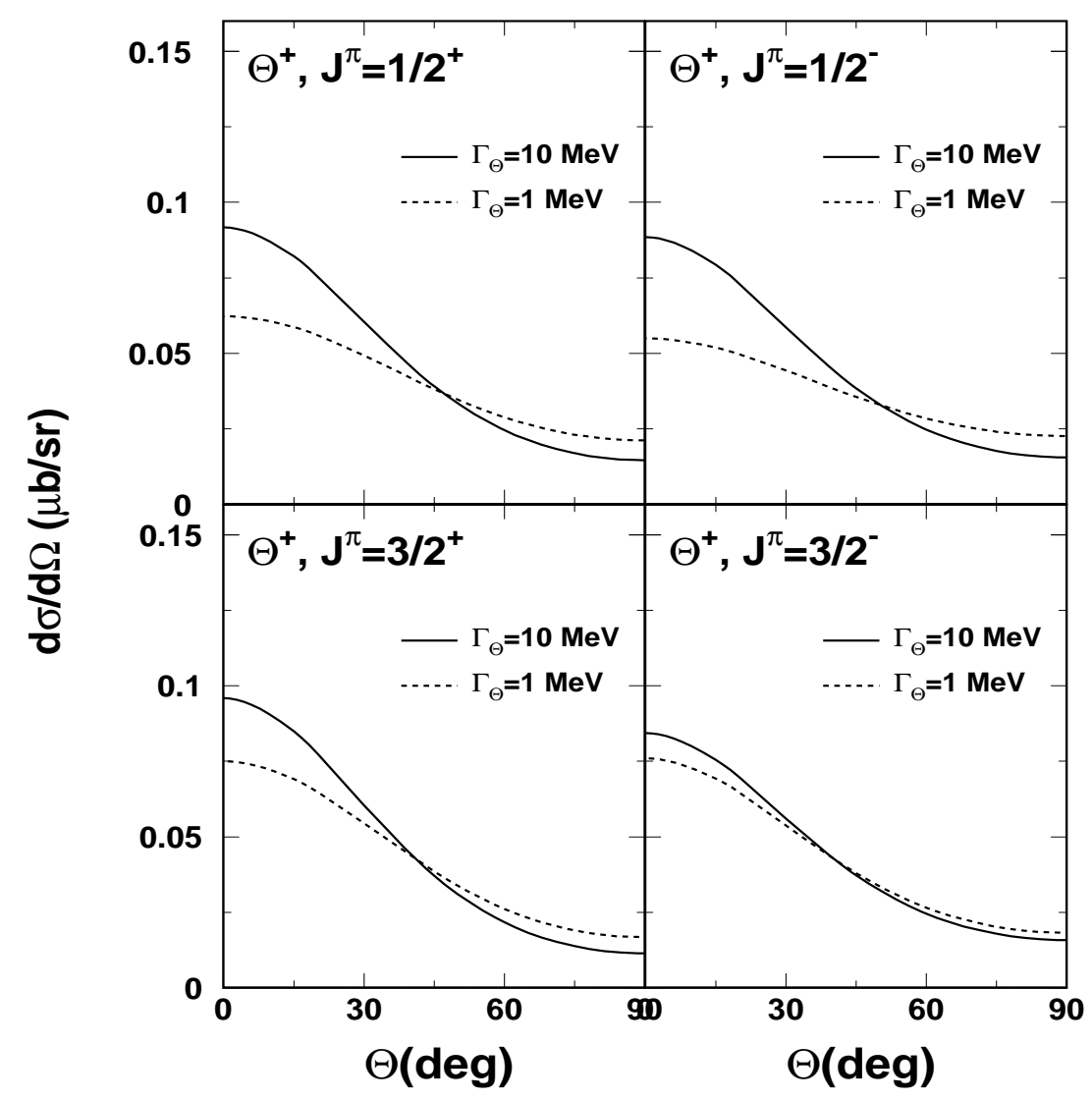

FIG. 2: Calculated angular distributions in the center-of-mass system for unpolarized beam and target for different spin-parity assignments of the $\Theta^{+}$states at an energy of $0.13 \mathrm{GeV}$ above threshold.

\section{THRESHOLD BEHAVIOR}

The threshold behavior has widely been discussed in refs. 14, 15, 16]. We summarize the consequences for the correlation parameters. The produced particles can only be in a state with relative orbital momentum $L=0$ since the higher partial waves are suppressed by the centrifugal barrier. The angular distribution is isotropic implying $A_{x z}=0$. Furthermore, parity conservation and Pauli principle imply that the total spin of the incoming protons is $S=0$ for positive parity of $\Theta^{+}$, and $S=1$ for negative parity.

If we set the polarization vectors $\mathbf{P}_{1}=\mathbf{P}_{2}$ and average over the direction of the polarization vectors we obtain from Eq. (15) the averaged integrated cross section

$$
\langle\sigma\rangle=\sigma_{0}\left(1+\frac{1}{3} P^{2}\left(A_{x x}+A_{y y}+A_{z z}\right)\right) .
$$

The same procedure can be applied to the polarization operator in Eq. (13) leading to

$$
\langle\sigma\rangle \sim 1+\frac{1}{3} P^{2}\left(\vec{\sigma}_{1} \vec{\sigma}_{2}\right)
$$

Comparing these two last equations one arrives at the relation $A_{x x}+A_{y y}+A_{z z}=-3(1)$ for $S=0(1)$, i. e. for positive (negative) parity of $\Theta^{+}$. 


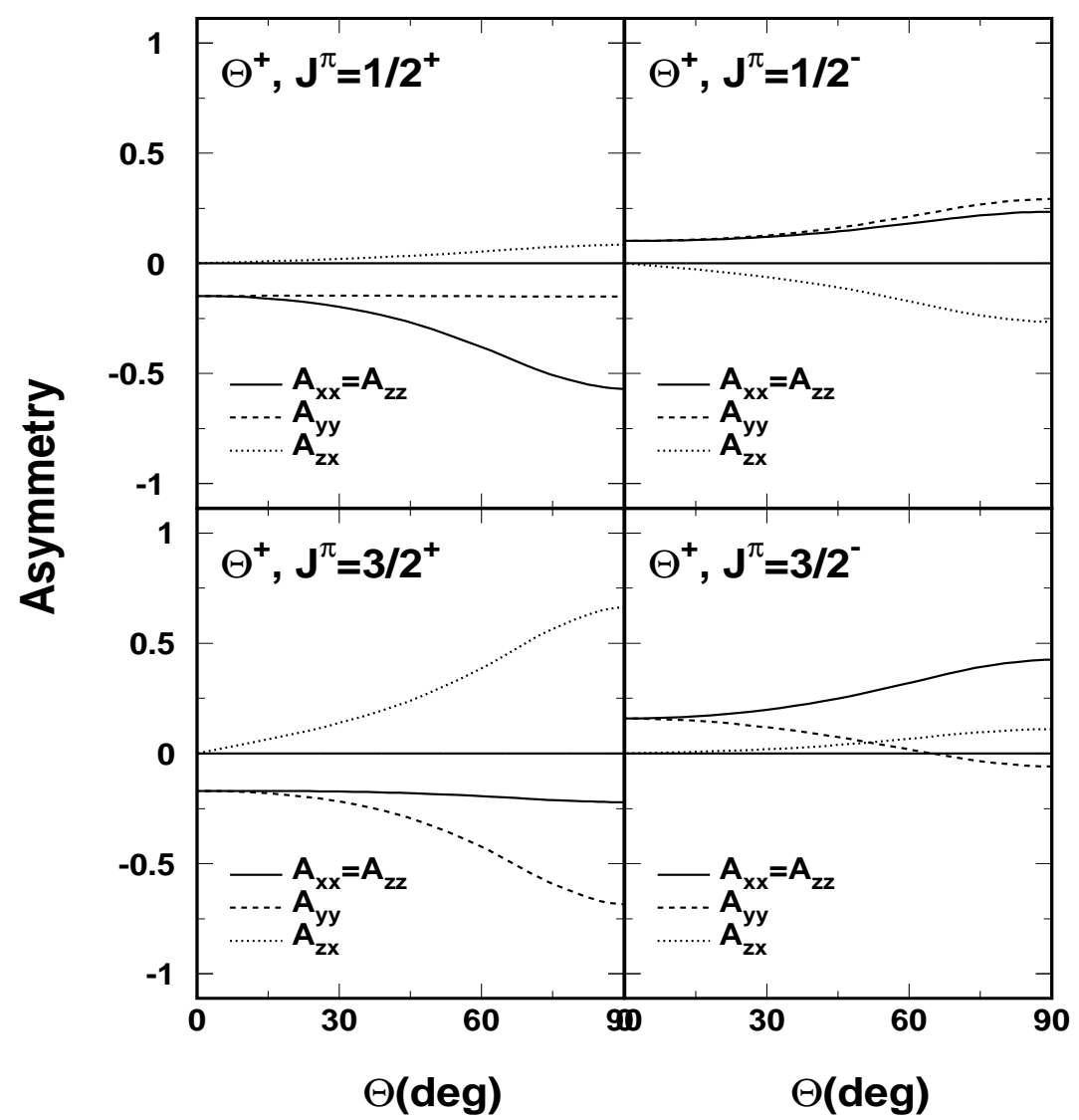

FIG. 3: Calculated correlation parameters as a function of the polar angle $\Theta$ for different assumptions on the spin-parity assignments of the $\Theta^{+}$particle at an excess energy of $0.13 \mathrm{GeV}$.

This causes the relation $A_{x x}=A_{y y}=A_{z z}=-1$ in the former case, while in the latter only the relations $A_{z z}=$ $1-A_{y y}-A_{x x}$ and $A_{y y}=A_{x x}$ can be derived in a model independent way since in this case different spin projections contribute to the $T$ matrix. For negative parities one obtains $A_{x x}=A_{y y}=A_{z z}=\frac{1}{3}$ if the kaon exchange of Eqs. (517) is used. But in general the coefficients depend on the parameters of the interaction used.

\section{RESULTS}

\section{A. $K$ exchange}

Here we consider only the $K$ exchange and calculate the differential cross section for the various possibilities of the $J^{\pi}$ assignments. We also investigate the effect of the width of the $\Theta^{+}$assuming values of $10 \mathrm{MeV}$ and $1 \mathrm{MeV}$ which are related to corresponding values of the coupling constants given in Eqs. (3] 810). As a constraint we vary the cut-off parameter $\Lambda$ such that we obtain a cross section of about $0.4 \mu \mathrm{b}$ at an excess energy of $\Delta E=\sqrt{s}-\sqrt{s_{\text {thr }}}$ $=0.13 \mathrm{GeV}$ above threshold. This value corresponds to the result obtained in a recent COSY measurement [9]. The values fulfilling this requirement are given in Table @

In Fig. 2 2 we present the angular distribution $(d \sigma / d \Omega)_{0}$ for unpolarized protons in the forward region. The cut-off parameter influences the shape of the angular distribution. A strong cut-off formfactor (small $\Lambda$ value) leads to a 


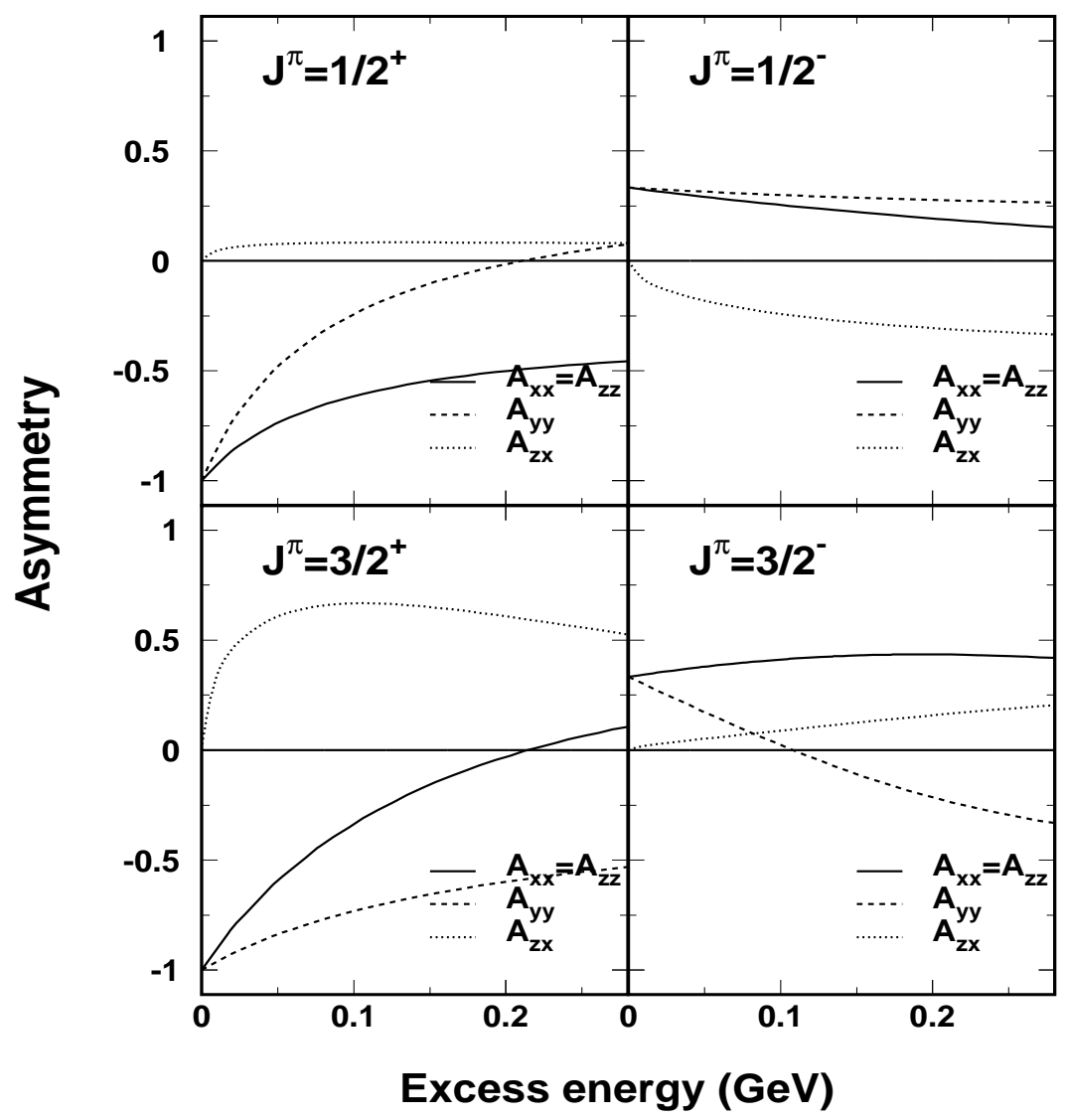

FIG. 4: Spin correlation parameters at 90 degrees in the center-of-mass system as a function of the excess energy $\sqrt{s}-\sqrt{s_{\text {thr }}}$ for different assumptions on the spin-parity assignments of the $\Theta^{+}$particle.

rather pronounced maximum in forward and backward direction.

In Fig. 3 we show the correlation parameters $A_{i i}$ in Eq. (15). These parameters are independent of the used formfactor. Most of them reach their maximum value at $90^{\circ}$. The side-side correlation parameter $A_{x x}$ and the longitudinal correlation parameter $A_{z z}$ coincide and $A_{z x}=-A_{x z}$ holds. Comparing the calculations with the opposite parities one recognizes that the transverse correlations $A_{y y}$ and $A_{x x}$ correlate their signs in coincidence with the assumed parity of the $\Theta^{+}$state. Measuring these coefficients could give a unique signal for the determination of the parity as was already found in refs. 14, 15, 16. A large positive normal-long correlation parameter $A_{z x}$ and a negative normal-normal correlation could signalize the $J^{\pi}=3 / 2^{+}$.

The energy dependence of the correlation parameters is shown in Fig. 14 It is seen that the characteristics of the threshold extend up to $50 \mathrm{MeV}$ a value that has also been estimated in ref. [14].

\section{B. $K^{*}$ exchange}

Now we investigate the effect of additionally including the $K^{*}$ exchange into the interaction. We treat the coupling constants in the Lagrangians (1112) as free parameters. To reduce the parameter space we choose the tensor coupling $\kappa=0$. This is not completely unrealistic, see [13]. Furthermore we relate the cut-off parameter to that of the $K$ exchange via $\Lambda_{K^{*}}=\Lambda_{K}+m_{K^{*}}-m_{K}$. Then we vary the values of the parameters $g^{*}$ and $f^{*}$ such that the cross 


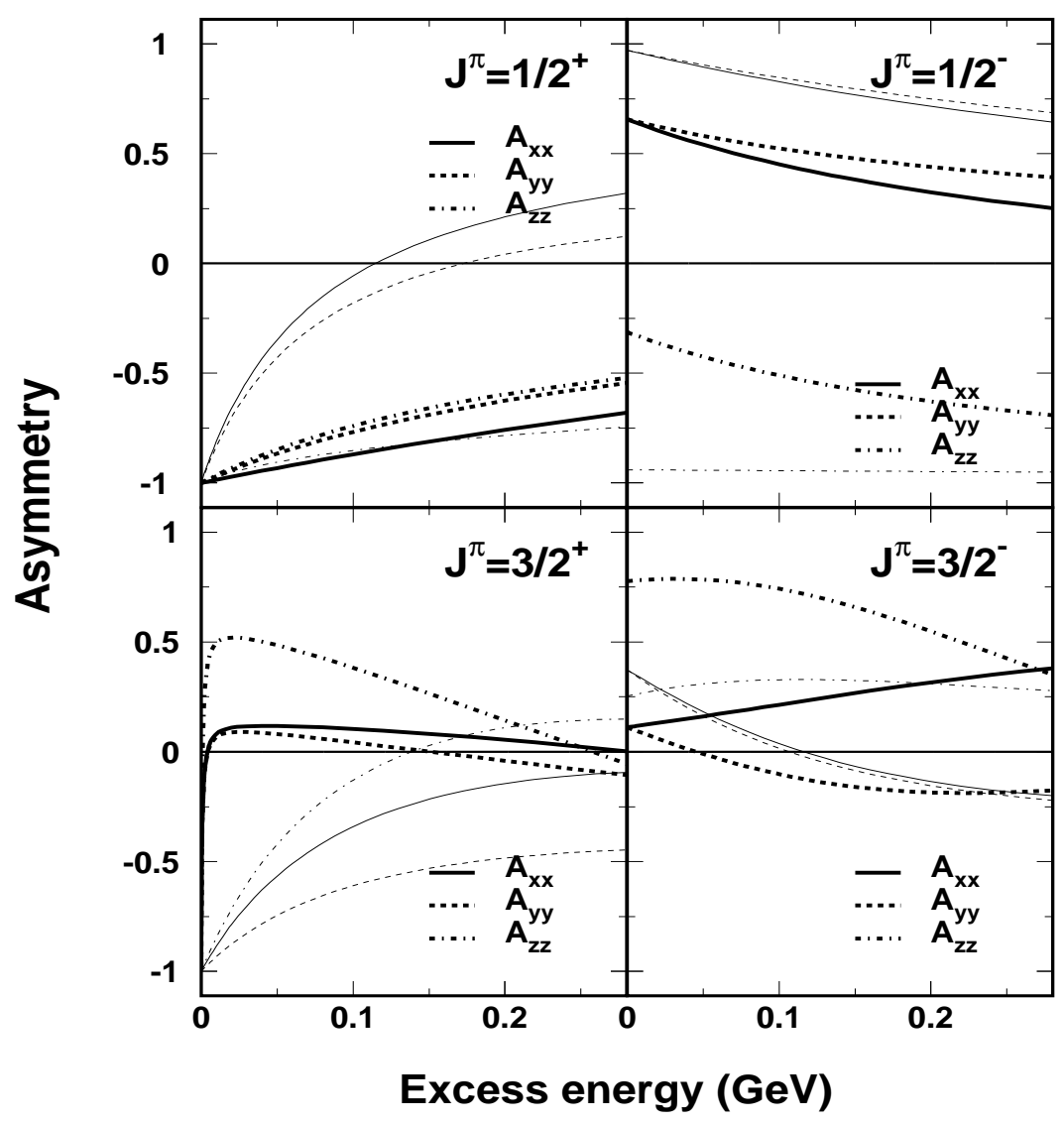

FIG. 5: Spin correlation parameters at 90 degrees as a function of the excess energy for a combination of $K$ and $K^{*}$ exchange. The thick (thin) lines present calculations with positive (negative) coupling strengths of the $K^{*}$ exchange given in Table

section of $0.4 \mu \mathrm{b}$ is increased to a maximum value of $0.8 \mu \mathrm{b}$. We made calculations with both possibilities of the signs of the coupling constants $f^{*}$ or $g^{*}$ leading to constructive or destructive interference between $K$ and $K^{*}$ exchange. The last two columns in Table \give the two possible values for the coupling coefficients.

In Fig. [5] we show the energy dependence of the spin correlation parameters $A_{i i}$. Comparing Figs. 4 and [5] one recognizes that the correlation parameters depend stronger on energy if the $K^{*}$ exchange has been included. The coefficients $A_{x x}$ and $A_{z z}$ do not coincide anymore. In particular $A_{x x}$ and $A_{y y}$ of the $1 / 2^{+}$state already change their signs at an excess energy of $100 \mathrm{MeV}$. A more drastic change of these coefficients is seen for the $3 / 2^{+}$state. This seems to contradict the estimates made in ref. 14]. The reason for this effect lies in the strong destructive interference between the interaction Lagrangians which reduces the cross section much stronger than one expects from the $\sqrt{\Delta E}$ threshold behavior. The constructive interference (thin lines, $f^{*}=-0.9$ ) does not show this strong energy dependence and agrees well with the estimates of the behavior of the threshold region given in ref. 14].

The behavior of the correlation coefficients for the negative states depends sensitively on the interaction used as can be seen by comparing Figs. 4 and 5 To identify the parity needs therefore the measurement of all three coefficients $A_{i i}$. 


\section{CONCLUSION}

We have analyzed the asymmetry of the angular distribution for $\Theta^{+}$pentaquark production in collisions of polarized protons. Using a variety of different interactions it was found that the characteristic threshold signals survive at energies up to $50 \mathrm{MeV}$ above threshold. Thus such measurements are a useful tool to determine spin and parity of the $\Theta^{+}$particle. In rare cases a more rapid change of the correlation parameters has been found which is accompanied with a stronger energy dependence than the expected $\sqrt{\Delta E}$ behavior of the cross section.

\section{Acknowledgments}

This research was supported by German BMBF grant 06DR121, the DAAD-MTA scientific exchange program between Germany and Hungary, and the National Fund for Scientific Research of Hungary, OTKA T047347.

[1] T. Nakano et al., (LEPS Collaboration), Phys. Rev. Lett. 91, 012002 (2003).

[2] V.V. Barnim et al. (DIANA Collaboration), Phys. Atom. Nucl. 66, 1715 (2003), Yad. Fiz. 66, 1763 (2003).

[3] S. Stepanyan et al. (CLAS Collaboration), Phys. Rev. Lett. 91, 252001 (2003).

[4] V. Kubarovsky et al. (CLAS Collaboration), Phys. Rev. Lett. 92, 032001 (2004).

[5] J. Barth et al. (SAPHIR Collaboration), hep-ex/0307083

[6] A. Airapetian et al. (HERMES Collaboration), Phys. Lett. B 585, 213 (2004)

[7] S. Chekanov et al. (ZEUS Collaboration), Phys. Lett. B 591, 7 (2004)

[8] D. Diakonov, V. Petrov, and M.V. Polyakov, Z. Phys. A 359, 305 (1997).

[9] M. Abdel-Bary et al. (COSY collaboration), Phys. Lett. B 595, 127 (2004).

[10] S.I. Nam, A. Hosaka, and. H.C. Kim, hep-ph/0403009

[11] W. Liu and C. M. Ko, Phys. Rev. C 68, 045203 (2003).

[12] K. Nakayama and K. Tsushima, Phys. Lett. B 583, 269, (2004)

[13] F.E. Close and Q. Zhao, Phys. Lett. B 590, 176 (2004).

[14] C. Hanhart et al., Phys. Lett. B 590, 39 (2004).

[15] S.I. Nam, A. Hosaka, and. H.C. Kim, hep-ph/0401074

[16] A.W. Thomas, K. Hicks and A. Hosaka, Prog. Theor. Phys. 111, 291 (2004)

[17] Yu.N. Uzikov, Phys.Lett. B 595, 277 (2004).

[18] M.P. Rekalo and E. Tomasi-Gustafson, Phys.Lett. B 591, 225 (2004).

[19] C.H. Li and C.M. Ko, Nucl. Phys. A 712, 110 (2002).

[20] Y. Oh, H. Kim, S.H. Lee, Phys.Rev. D69 (2004) 094009.

[21] W. Liu, C.M. Ko, V. Kubarovsky, Phys.Rev. C69 (2004) 025202.

[22] J. Bystricky, F. Lehar, and P. Winternitz, Journal de Physique 39, 1 (1978).

[23] H.O. Meyer et al., Phys. Rev. C 63, 064002 (2001). 